\title{
On the sign of fermion-mediated interactions
}

\author{
Qing-Dong Jiang $\odot$ \\ Department of Physics, Stockholm University, SE-106 91 Stockholm, Sweden
}

(Received 18 October 2020; accepted 26 February 2021; published 10 March 2021)

\begin{abstract}
We develop a unified framework for understanding the sign of fermion-mediated interactions by exploiting the symmetry classification of Green's functions. In particular, we establish a theorem regarding the sign of fermion-mediated interactions in systems with chiral symmetry. The strength of the theorem is demonstrated within multiple examples with an emphasis on electron-mediated interactions in materials.
\end{abstract}

DOI: 10.1103/PhysRevB.103.L121107

Introduction. That the exchange of a particle can produce a force is one of the most remarkable conceptual advances in physics. Each of the fundamental interactions has an associated bosonic force carrier: For example, photons mediate Coulomb interactions and gravitons mediate gravitational interactions. Given these boson-mediated interactions, one may naturally ask the following innocuous question: "Can fermions also mediate interactions?"

While the answer is "yes," there is an essential difference between boson-mediated interactions and fermion-mediated interactions. That is, due to the conservation of fermionic parity, fermions need to be exchanged at least twice to produce a force whereas bosons only need to be exchanged once (see the Feynman diagram in Fig. 1). As this Feynman diagram resembles that of the Casimir effect, fermion-mediated interactions are occasionally called fermionic Casimir effects in the literature [1]. In the case of one-boson-mediated interactions, the sign is uniquely determined by the spin of the exchanged particles: Exchanging a scalar, or a tensor, particle produces a universally attractive force, while exchanging a vector particle can produce a repulsive force between like charges [2]. However, unlike the bosonic case, a universal understanding of the sign of fermion-mediated interactions is currently lacking.

In this Letter, we study the sign of various fermionmediated interactions. In condensed-matter physics, electronmediated interactions were initially proposed to explain the ordering of adsorbates at surfaces [3,4], and were recently considered to be crucial for engineering the properties of novel materials such as graphene [5]. As an important mechanism for magnetic ordering, the RudermanKittel-Kasuya-Yosida (RKKY) interaction is another example of electron-mediated interactions [6]. In the cold-atom area, fermion-mediated interactions have received extensive

Published by the American Physical Society under the terms of the Creative Commons Attribution 4.0 International license. Further distribution of this work must maintain attribution to the author(s) and the published article's title, journal citation, and DOI. Funded by Bibsam. theoretical investigations [7] and were recently observed in experiments consisting of a mixture of bosonic and fermionic quantum gases [8]. In high-energy physics, fermion-mediated interactions are of particular relevance to the physics of neutron stars [9] and quark-gluon plasma [10]. Given the ubiquitous presence of fermion-mediated interactions, their sign is of both theoretical interest and practical significance [11-13]. For example, attractive fermion-mediated interactions could lead to new phases of matter such as supersolids [14].

By exploiting the symmetry classification of Green's functions $[15,16]$, we present a unified framework for understanding the sign of fermion-mediated interactions, eliminating some loopholes and resolving some controversies in the literature. The symmetry that plays the central role is called chiral symmetry, which is a combination of time-reversal symmetry and charge-conjugation symmetry. The associated chirality corresponds to whether an operator is even or odd under the action of the chiral symmetry. Specifically, in systems with chiral symmetry, we show that the sign of fermion-mediated interactions $U_{12}$ between objects $X_{1}$ and $X_{2}$ is given by the simple rule

$$
\operatorname{sgn}\left(\mathrm{U}_{12}\right)=(-1)^{\eta} \chi_{1} \chi_{2}
$$

Here, $\chi_{1(2)}= \pm 1$ is the chirality of the object $\mathrm{X}_{1(2)} ; \eta=0$ or 1 depending on the strength of scattering potentials being strong or weak. Between objects with the same chirality, a strong scattering potential leads to repulsion, whereas a weak scattering potential leads to attraction. By sharp contrast, between objects with opposite chiralities, a strong scattering potential leads to attraction, whereas a weak scattering potential leads to repulsion.

The rest of the Letter is organized as follows. We first derive a nonperturbative expression of fermion-mediated interactions involving Matsubara Green's functions and scattering matrices. Then, we show how nonspatial (local) symmetries constrain the Green's functions and scattering matrices of a general interacting system. With the above preparation, we establish a theorem regarding the sign of fermion-mediated interactions. Finally, we demonstrate the power of our theorem by giving a number of examples with an emphasis on electron-mediated interactions in materials. 
Field theory of fermion-mediated interactions. To set the stage, we now present a unified expression for fermionmediated interactions. While various expressions for fermionmediated interactions exist in the literature [11,12,17], a universal, and nonperturbative, derivation is not entirely trivial. A related path-integral approach has been used to study the Casimir effect in Refs. [18,19].

Consider two objects $\mathrm{X}_{i}(i=1,2)$ embedded at the positions $\mathbf{x}_{i}$ in a fermionic environment, and introduce localized potential operators $\hat{\mathcal{V}}_{i}$ to describe the scattering effect of $\mathrm{X}_{i}$. We consider bilinear coupling $\bar{\psi} \hat{\mathcal{V}}_{i} \psi$ between the operators $\hat{\mathcal{V}}_{i}$ and the mediating fermionic fields $(\bar{\psi}, \psi)$; thus the effective Euclidean action reads (we use units $\hbar=c=1$ )

$$
\mathcal{S}=-\sum_{n} \int d \mathbf{x} \bar{\psi}_{n}(\mathbf{x})\left(\hat{\mathcal{G}}^{-1}-\hat{\mathcal{V}}_{1}-\hat{\mathcal{V}}_{2}\right) \psi_{n}(\mathbf{x}),
$$

where $\hat{\mathcal{G}}=\left(i \omega_{n}+\hat{H}\right)^{-1}$ represents the Matsubara Green's function of the fermionic host. The partition function of the whole system is $\mathcal{Z}=\int \mathcal{D} \bar{\psi} \mathcal{D} \psi \exp (-\mathcal{S})$. In the absence of $\mathrm{X}_{i}$, the partition function $\mathcal{Z}_{0}$ can be obtained from $\mathcal{Z}$ by setting $\hat{\mathcal{V}}_{i}=0$. Consequently, the change of the energy due to the introduction of $\mathrm{X}_{i}$ can be formally obtained from the reduced partition function

$$
\begin{aligned}
\mathcal{E} & =-\frac{1}{\beta} \ln \frac{\mathcal{Z}}{\mathcal{Z}_{0}} \\
& =-\frac{1}{\beta} \ln \operatorname{det}\left[1-\mathcal{G}\left(\mathbf{x}, \mathbf{x}^{\prime}\right) \mathcal{V}_{1}-\mathcal{G}\left(\mathbf{x}, \mathbf{x}^{\prime}\right) \mathcal{V}_{2}\right]
\end{aligned}
$$

where $\mathcal{G}\left(\mathbf{x}, \mathbf{x}^{\prime}\right)=\left\langle\mathbf{x}|\hat{\mathcal{G}}| \mathbf{x}^{\prime}\right\rangle, \mathcal{V}_{i}=\left\langle\mathbf{x}^{\prime}\left|\hat{\mathcal{V}}_{i}\right| \mathbf{x}^{\prime}\right\rangle \delta_{\mathbf{x}^{\prime}, \mathbf{x}_{i}}$, and $\beta$ is the inverse temperature. Note that the total energy $\mathcal{E}$ contains three parts: the self-energies of $\mathrm{X}_{1}$ and $\mathrm{X}_{2}$ and the mutual interaction between them. Therefore, to obtain the interaction energy, we need to subtract the self-energy contribution that does not depend on the relative positions of $X_{1}$ and $X_{2}$. For this purpose, it is convenient to put the energy in a matrix form:

$$
\mathcal{E}=-\frac{1}{\beta} \ln \operatorname{det}\left(\begin{array}{cc}
1-\mathcal{G}_{11} \mathcal{V}_{1} & -\mathcal{G}_{12} \mathcal{V}_{2} \\
-\mathcal{G}_{21} \mathcal{V}_{1} & 1-\mathcal{G}_{22} \mathcal{V}_{2}
\end{array}\right)
$$

Here, $\mathcal{G}_{12} \equiv \mathcal{G}\left(i \omega_{n}, \mathbf{r}\right)$ and $\mathcal{G}_{21} \equiv \mathcal{G}\left(i \omega_{n},-\mathbf{r}\right)$ are the Matsubara Green's functions linking $\mathrm{X}_{1}$ with $\mathrm{X}_{2} ; \mathcal{G}_{11}=\mathcal{G}_{22} \equiv$ $\mathcal{G}\left(i \omega_{n}, 0\right)$ are local Matsubara Green's functions; $\mathbf{r}=\mathbf{x}_{2}-\mathbf{x}_{1}$ denotes the relative distance between $\mathrm{X}_{1}$ and $\mathrm{X}_{2}$; and $\omega_{n}=$ $(2 n+1) \pi \beta^{-1}$ are fermionic Matsubara frequencies. After subtracting the self-energies, i.e., the diagonal contribution of the matrix, we obtain the universal formula for two-particle exchange interactions,

$$
\mathrm{U}_{12}=-\frac{1}{\beta} \sum_{n} \ln \operatorname{det}\left(1-\mathcal{G}_{12} T_{2} \mathcal{G}_{21} T_{1}\right),
$$

where $T_{1}=\mathcal{V}_{1}\left(1-\mathcal{G}_{11} \mathcal{V}_{1}\right)^{-1}$ and $T_{2}=\mathcal{V}_{2}\left(1-\mathcal{G}_{22} \mathcal{V}_{2}\right)^{-1}$ represent the scattering matrices for $X_{1}$ and $X_{2}$ [20]. Note that Eq. (5) is derived without using any perturbative expansion, and thus it applies to strong scattering potentials. If the fermionic host is weakly correlated, $\mathcal{G}_{12}$ and $\mathcal{G}_{21}$ represent the renormalized Matsubara Green's functions with interactions encoded in their self-energy parts. To the lowest-order expansion, $\mathrm{U}_{12}=\frac{1}{\beta} \sum_{n} \mathcal{G}_{12} T_{2} \mathcal{G}_{21} T_{1}$ represents

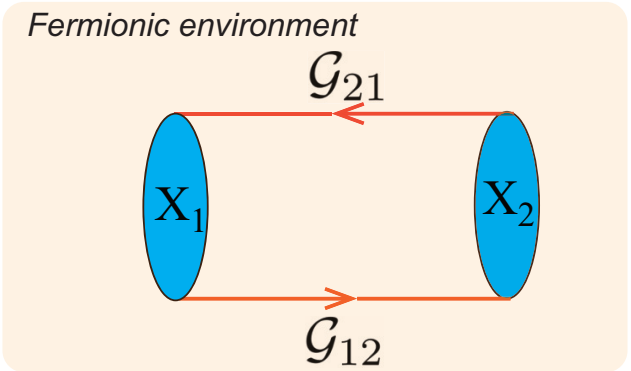

FIG. 1. Feynman diagram for fermion-mediated interactions. The red lines represent fermionic Green's functions (propagators) $\mathcal{G}_{12}$ and $\mathcal{G}_{21}$ that connect $\mathrm{X}_{1}$ and $\mathrm{X}_{2}$. Since two Green's functions are involved in the Feynman diagram, we say that objects $X_{1}$ and $\mathrm{X}_{2}$ interact with each other by exchanging fermions twice. The blue bubbles represent the scattering matrices of $X_{1}$ and $X_{2}$, the form of which will be given in the text.

a two-fermion-exchange interaction, as illustrated in Fig. 1. One could obtain the zero-temperature formula by replacing $\frac{1}{\beta} \sum_{n}$ with $\int \frac{d \xi}{2 \pi}$ ( $\xi$ is the imaginary frequency) in Eq. (5), which agrees with the result in the literature [11,12].

Symmetry classification of Green's functions. We now present one more ingredient - the symmetry classification of Green's functions-aiming at a universal theorem for the sign of fermion-mediated interactions. In recent years, the classification of Green's functions under nonspatial symmetries, namely time-reversal symmetry, charge-conjugation (particle-hole) symmetry, and chiral symmetry, has been used to classify topological phases of correlated fermions [15]. For clarity, we start with the symmetry classification of noninteracting Hamiltonians [16] and then derive the symmetry classification of Matsubara Green's functions which turns out to be particularly useful when interactions are present.

Consider a general system of noninteracting fermions described by the Hamiltonian $\hat{H}=\sum_{\alpha \beta} \psi_{\alpha}^{\dagger} \mathcal{H}_{\alpha \beta} \psi_{\beta}$ with the fermion creation and annihilation operators satisfying canonical anticommutation relations $\left\{\psi_{\alpha}, \psi_{\beta}^{\dagger}\right\}=\delta_{\alpha \beta}$. Here, the indices $\alpha, \beta$ refer to relevant degrees of freedom, such as lattice sites, spins, layers, etc.; and $\mathcal{H}$, the first quantized Hamiltonian, is a complex matrix. Under the constraints of time-reversal symmetry and charge-conjugation symmetry, the Hamiltonian matrix $\mathcal{H}$ satisfies the following conditions,

$$
\begin{aligned}
& U_{T}^{\dagger} \mathcal{H}^{*}(\mathbf{k}) U_{T}=\mathcal{H}(-\mathbf{k}), \\
& U_{C}^{\dagger} \mathcal{H}^{*}(\mathbf{k}) U_{C}=-\mathcal{H}(-\mathbf{k}),
\end{aligned}
$$

where $U_{T}$ and $U_{C}$ are the corresponding unitary matrices. It is crucial to consider an additional discrete symmetry, the chiral symmetry, being the product of time-reversal symmetry and charge-conjugation symmetry. The chiral symmetry imposes an additional condition on the Hamiltonian matrix

$$
U_{S}^{\dagger} \mathcal{H}(\mathbf{k}) U_{S}=-\mathcal{H}(\mathbf{k})
$$

with the chiral matrix $U_{S}=U_{T}^{*} U_{C}$. Notice that the chiral symmetry can be present in cases where neither time-reversal nor charge-conjugation symmetry is present. The above symmetry constraints lead to the tenfold classification of topological insulators and superconductors [16]. 
Equipped with the above Hamiltonian formalism, we now proceed to present the symmetry classification of the Green's functions. We are interested in the Matsubara Green's functions defined as $\mathcal{G}\left(i \omega_{n}, \mathbf{k}\right)=\left[i \omega_{n}-\mathcal{H}(\mathbf{k})\right]^{-1}$. According to Eqs. (6a), (6b), and (7), time-reversal symmetry and charge-conjugation symmetry set the following conditions for Matsubara Green's functions, i.e.,

$$
\begin{aligned}
& U_{T} \mathcal{G}\left(i \omega_{n}, \mathbf{k}\right) U_{T}^{\dagger}=\frac{1}{i \omega_{n}-\mathcal{H}^{*}(-\mathbf{k})}=\mathcal{G}^{*}\left(-i \omega_{n},-\mathbf{k}\right), \\
& U_{C} \mathcal{G}\left(i \omega_{n}, \mathbf{k}\right) U_{C}^{\dagger}=\frac{1}{i \omega_{n}+\mathcal{H}^{*}(-\mathbf{k})}=-\mathcal{G}^{*}\left(i \omega_{n},-\mathbf{k}\right) .
\end{aligned}
$$

Furthermore, chiral symmetry implies that the Matsubara Green's function should fulfill the condition

$$
U_{S} \mathcal{G}\left(i \omega_{n}, \mathbf{k}\right) U_{S}^{\dagger}=-\mathcal{G}\left(-i \omega_{n}, \mathbf{k}\right),
$$

consistent with Eqs. (8a) and (8b). Finally, regardless of whether or not interactions are present, the Hermicity of the Hamiltonian ensures that

$$
\mathcal{G}\left(i \omega_{n}, \mathbf{k}\right)=\mathcal{G}^{\dagger}\left(-i \omega_{n}, \mathbf{k}\right)
$$

As a result, the combination of chiral symmetry and Hermicity leads to the momentum-space condition

$$
U_{S} \mathcal{G}\left(i \omega_{n}, \mathbf{k}\right) U_{S}^{\dagger}=-\mathcal{G}^{\dagger}\left(i \omega_{n}, \mathbf{k}\right),
$$

which, after Fourier transform, yields the real-space expression

$$
U_{S} \mathcal{G}\left(i \omega_{n}, \mathbf{r}\right) U_{S}^{\dagger}=-\mathcal{G}^{\dagger}\left(i \omega_{n},-\mathbf{r}\right),
$$

which is an essential ingredient in the proof of our theorem. While the above Eqs. (8a), (8b), and (9) are obtained from noninteracting Hamiltonians, a sophisticated field-operator approach shows that the above Green's function formalism also holds for interacting systems. Interested readers could also find the proof from the excellent works in Ref. [15].

The theorem. In systems with symmetry, it is often convenient to choose a chiral basis such that the chiral operator $U_{S}$ is diagonal, i.e., $U_{S}=\operatorname{diag}\left(\mathbb{1}_{n},-\mathbb{1}_{m}\right)$, where $\mathbb{1}_{n}$ and $\mathbb{1}_{m}$ are $n \times n$ and $m \times m$ identity matrices. The eigenvalue $+1(-1)$ denotes the chirality of the corresponding basis. One can then use the Pauli matrix $\tau_{z}$ to represent $U_{S}$ for each pair of basis states with opposite chiralities. (We use $\tau_{x, y, z}$ to represent Pauli matrices for general degrees of freedom, while reserving the notation $\sigma_{x, y, z}$ for real spin.) The Matsubara Green's function matrix in the pair chiral basis $(|\chi\rangle,|\bar{\chi}\rangle)$ reads

$$
\mathcal{G}\left(i \omega_{n}, \mathbf{r}\right)=\left(\begin{array}{ll}
\mathcal{G}^{\chi \chi}\left(i \omega_{n}, \mathbf{r}\right) & \mathcal{G}^{\chi \bar{\chi}}\left(i \omega_{n}, \mathbf{r}\right) \\
\mathcal{G}^{\bar{\chi} \chi}\left(i \omega_{n}, \mathbf{r}\right) & \mathcal{G}^{\bar{\chi} \bar{\chi}}\left(i \omega_{n}, \mathbf{r}\right)
\end{array}\right)
$$

where the indices $\chi=-\bar{\chi}= \pm 1$ represent chiralities of the corresponding basis. Apparently, the diagonal components of the Green's function connect objects with the same chirality, whereas the off-diagonal components connect objects with opposite chiralities. By substituting the Matsubara Green's function and $U_{S}=\tau_{z}$ into Eq. (12), we obtain the crucial conditions required by the chiral symmetry,

$$
\begin{aligned}
& \mathcal{G}^{\chi \chi}\left(i \omega_{n}, \mathbf{r}\right)=-\mathcal{G}^{\chi \chi *}\left(i \omega_{n},-\mathbf{r}\right), \\
& \mathcal{G}^{\chi \bar{\chi}}\left(i \omega_{n}, \mathbf{r}\right)=\mathcal{G}^{\bar{\chi} \chi *}\left(i \omega_{n},-\mathbf{r}\right) .
\end{aligned}
$$

If two objects have the same chirality, the connecting Matsubara Green's functions satisfy $\mathcal{G}_{12} \equiv \mathcal{G}^{\times x}\left(i \omega_{n}, \mathbf{r}\right)=$ $-\mathcal{G}_{21}^{*} \equiv-\mathcal{G}^{\chi \chi *}\left(i \omega_{n},-\mathbf{r}\right)$. By contrast, if two objects have opposite chiralities, the connecting Matsubara Green's functions satisfy $\mathcal{G}_{12} \equiv \mathcal{G}^{\chi \bar{x}}\left(i \omega_{n}, \mathbf{r}\right)=\mathcal{G}_{21}^{*} \equiv \mathcal{G}^{\bar{\chi}} \chi^{*}\left(i \omega_{n},-\mathbf{r}\right)$. As indicated by Eq. (5), the sign of the fermion-mediated interaction is identical to the sign of the product $\mathcal{G}_{12} T_{2} \mathcal{G}_{21} T_{1}$ [21]. Due to the expression of the scattering matrices [below Eq. (5)], two possibilities can be distinguished:

(1) In the limit of a strong potential $\left(\mathcal{V}_{i} \rightarrow \infty\right)$, the scattering matrices $T_{1}=T_{2}=\mathcal{G}^{-1}\left(i \omega_{n}, 0\right)$ are purely imaginary according to Eq. (14a), and therefore $T_{1} T_{2}<0$. It is then straightforward to obtain the sign of fermion-mediated interactions in the following cases:

(i) Between two objects with the same chirality, the fermion-mediated interactions are always repulsive because

$$
\begin{aligned}
\operatorname{sgn}\left(\mathrm{U}_{12}\right) & =\operatorname{sgn}\left(\mathcal{G}_{12} T_{2} \mathcal{G}_{21} T_{1}\right) \\
& =-\operatorname{sgn}\left(\mathcal{G}_{21}^{*} T_{2} \mathcal{G}_{21} T_{1}\right)>0 .
\end{aligned}
$$

(ii) Between two objects with opposite chiralities, the fermion-mediated interactions are always attractive due to

$$
\begin{aligned}
\operatorname{sgn}\left(\mathrm{U}_{12}\right) & =\operatorname{sgn}\left(\mathcal{G}_{12} T_{2} \mathcal{G}_{21} T_{1}\right) \\
& =\operatorname{sgn}\left(\mathcal{G}_{21}^{*} T_{2} \mathcal{G}_{21} T_{1}\right)<0 .
\end{aligned}
$$

(2) In the limit of a weak potential $\left(\mathcal{V}_{i} \rightarrow 0\right)$, the scattering matrices $T_{i}=\mathcal{V}_{i}$ are purely real, and $T_{1} T_{2}>0$. Hence, the signs of fermion-mediated interactions are opposite to the case of strong potential: Between two objects with the same (opposite) chirality, the fermion-mediated interactions are always attractive (repulsive).

As the above results can be conveniently summarized in Eq. (1), we have thus established the theorem for the sign of fermion-mediated interactions. The above analysis indicates that one could change the sign of fermion-mediated interactions by tuning the strength of the scattering potentials. To demonstrate the power of this theorem, we now explore a number of examples with an emphasis on electron-mediated interactions in materials.

Electron-mediated interactions in noninteracting systems. As our first example, let us consider electron-mediated interactions between two adatoms embedded in monolayer graphene. The physics of single-layer graphene is captured by the continuum low-energy Hamiltonian $\mathcal{H}_{\mathrm{SLG}}=$ $v_{F}\left(k_{x} \tau_{x}+k_{y} \tau_{y}\right)$, where $\tau_{x, y, z}$ represent the Pauli matrices in the sublattice basis, and $v_{F}$ is the Fermi velocity [22]. One can easily identify the chiral transformation matrix $U_{S}=\tau_{z}$. Therefore, without a further detailed calculation, we can apply the theorem to graphene. In the strong-impurity limit [23], adatoms residing on the same (different) sublattices repel (attract), whereas in the weak-impurity limit, impurities residing on the same (different) sublattices attract (repel). This is consistent with detailed calculations carried out in Refs. [11,12] One can consider more general Hamiltonians such as $\mathcal{H}=v_{F}\left(k_{x}^{n} \tau_{x}+k_{y}^{m} \tau_{y}\right)$, where $m$ and $n$ can be arbitrary odd numbers. As the chiral matrix for this Hamiltonian is still $U_{S}=\tau_{z}$, our theorem also applies to this model.

Our second example deals with the electron-mediated interactions in the Bernal-stacked bilayer graphene, the unit cell of which includes $A_{1}$ and $B_{1}$ atoms on layer 1 and $A_{2}$ and $B_{2}$ 
atoms on layer 2. Expressed in the basis $\left(\psi_{A 1}, \psi_{A 2}, \psi_{B 1}, \psi_{B 2}\right)$ (here subindices denote the lattice type), the low-energy effective Hamiltonian reads [24]

$$
\mathcal{H}_{\mathrm{BLG}}=\left(\begin{array}{cc}
0 & \mathcal{D}^{*}(-\mathbf{r}) \\
\mathcal{D}(\mathbf{r}) & 0
\end{array}\right), \quad \mathcal{D}(\mathbf{r})=\left(\begin{array}{cc}
-i \partial_{+} & \gamma \\
0 & -i \partial_{+}
\end{array}\right),
$$

where $\partial_{ \pm}=\partial_{x} \pm i \partial_{y}$ and $\gamma$ denotes the interlayer coupling; we set the Fermi velocity $v_{F}=1$. It can be verified that this Hamiltonian preserves chiral symmetry with the chiral matrix $U_{S}=\tau_{z} \otimes \mathbb{1}$, which indicates that sublattices $\left(\mathrm{A}_{1}, \mathrm{~A}_{2}\right)$ have positive chirality while sublattices $\left(B_{1}, B_{2}\right)$ have negative chirality. Accordingly, regardless of the layer index, the electron-mediated interactions are always repulsive (attractive) between impurities on the same type of sublattices (i.e., AA or BB) in the strong- (weak-) impurity limit. In contrast, when impurities reside on different sublattices (i.e., $\mathrm{AB})$, the electron-mediated interactions are always attractive (repulsive) in the strong- (weak-) impurity limit.

One may also apply the theorem to study the twisted bilayer model, which has generated considerable interest recently. A specific chiral-symmetric model [25] that supports exactly flat bands has a similar form as Eq. (17) but with a different $\mathcal{D}$ matrix,

$$
\mathcal{D}_{\mathrm{tBLG}}(\mathbf{r})=\left(\begin{array}{cc}
-v_{F} i \partial_{+} & \mathcal{F}(\theta, \mathbf{r}) \\
\mathcal{F}(\theta,-\mathbf{r}) & -v_{F} i \partial_{+}
\end{array}\right),
$$

where the moiré potential $\mathcal{F}(\theta, \mathbf{r})$ is a function of the twist angle $\theta$ and position $\mathbf{r}$. Regardless of the specific form of $\mathcal{F}(\theta, \mathbf{r})$, this model preserves chiral symmetry with the same chiral matrix as Bernal-stacked bilayer graphene. Consequently, the sign of an electron-mediated interaction in twisted bilayer model shares the identical feature with that in the Bernal-stacked bilayer graphene. The chiral-symmetry protected sign of fermion-mediated interactions could be of essential importance for understanding disorder-related physics in twisted bilayer models. We should, however, clarify that twisted bilayer materials in experiments may violate chiral symmetry due to the substrate or interactions.

Electron-mediated interaction in correlated systems. Our theorem is applicable to weakly interacting systems. We examine electron-mediated interactions in the Hubbard model defined on a bipartite lattice

$$
\hat{H}=\sum_{\langle i, j\rangle, \sigma} t_{i j} \hat{c}_{i, \sigma}^{\dagger} \hat{c}_{j, \sigma}-\mu \sum_{i} \hat{n}_{i \sigma}+U \sum_{i} \hat{n}_{i \uparrow} \hat{n}_{i \downarrow},
$$

where $\hat{c}_{i, \sigma}$ and $\hat{c}_{i, \sigma}^{\dagger}$ is the electron annihilation and creation operators at site $i$ with spin $\sigma=\uparrow$ or $\downarrow$ and $\hat{n}_{i \sigma}=\hat{c}_{i \sigma}^{\dagger} \hat{c}_{i \sigma}$. Here, the nearest-neighbor hopping matrix elements $t_{i j}=t_{j i}$ need to be real; $\mu$ and $U$ represent the chemical potential and on-site interaction strength, respectively. One can verify that the Hamiltonian is invariant at half filling $(\mu=U / 2)$ under the following chiral symmetry transformation: $\hat{\mathcal{S}} \hat{c}_{i \uparrow / \downarrow} \hat{\mathcal{S}}^{-1}=$ $\kappa(i) \hat{c}_{i \downarrow(\uparrow)}^{\dagger}, \hat{\mathcal{S}} \hat{c}_{i \uparrow(\downarrow)}^{\dagger} \hat{\mathcal{S}}^{-1}=\kappa(i) \hat{c}_{i \downarrow(\uparrow)}$, with $\kappa(i)=1$ for one of the sublattices and $\kappa(i)=-1$ for the other. It should be obvious that the same sublattices have the same chirality and different sublattices have opposite chiralities. We assume that $U$ is small enough for the ground state to preserve chiral symmetry so that our result Eq. (5) still holds. Without performing any perturbative calculation, we can apply our theorem to the correlated bipartite lattices: Fermion-mediated interactions are repulsive or attractive depending on the impurities located on the same or different sublattices. Our theorem can be applied to all weakly coupled models with chiral symmetry, such as Bogoliubov-de Gennes (BdG) systems with time-reversal symmetry [26] and quantum chromodynamics (QCD) at high density [27].

RKKY interactions in systems with chiral symmetry. A similar line of reasoning enables us to predict the sign of RKKY interactions in the presence of chiral symmetry: Magnetic moments with the same (opposite) chirality favor a ferromagnetic (antiferromagnetic) state. We demonstrate this statement by considering two magnetic moments $\left(\mathbf{S}_{1}\right.$ and $\left.\mathbf{S}_{2}\right)$ embedded in a fermionic system described by the effective action

$$
\begin{aligned}
\mathcal{S}= & -\sum_{n} \int d \mathbf{x} \bar{\psi}_{n}(\mathbf{x})\left[\mathcal{G}^{-1}-\frac{J}{2} \mathbf{S}_{1} \cdot \boldsymbol{\sigma} \delta\left(\mathbf{x}-\mathbf{x}_{1}\right)\right. \\
& \left.-\frac{J}{2} \mathbf{S}_{2} \cdot \boldsymbol{\sigma} \delta\left(\mathbf{x}-\mathbf{x}_{2}\right)\right] \psi_{n}(\mathbf{x}),
\end{aligned}
$$

with $\mathcal{G}$ being the Green's function of the host system and $J$ as the coupling constant between the impurity magnetic moment and the spin of electrons $\sigma_{\mu}(\mu=x, y, z)$ located at $\mathbf{x}_{1}$ and $\mathbf{x}_{2}$, respectively. Note that this action has the same form as Eq. (2) given the substitution $\hat{\mathcal{V}}_{i}=\mathbf{S}_{i} \cdot \boldsymbol{\sigma} \delta\left(\mathbf{x}-\mathbf{x}_{i}\right)$. According to Eq. (5), we then obtain the interaction energy of the nuclear spins (to the lowest order of $J$ ),

$$
\begin{aligned}
\mathrm{U}_{12} & =\frac{J^{2}}{4 \beta} \sum_{\mu, \nu, n} \operatorname{tr}\left[S_{1 \mu} \sigma_{\mu} \mathcal{G}\left(i \omega_{n}, \mathbf{r}\right) S_{2 \nu} \sigma_{\nu} \mathcal{G}\left(i \omega_{n},-\mathbf{r}\right)\right] \\
& =-J^{2} \alpha(\mathbf{r}) \mathbf{S}_{1} \cdot \mathbf{S}_{2},
\end{aligned}
$$

where the susceptibility is defined as $\alpha(\mathbf{r})=$ $-\frac{1}{2 \beta} \sum_{n} \mathcal{G}\left(i \omega_{n}, \mathbf{r}\right) \mathcal{G}\left(i \omega_{n},-\mathbf{r}\right)$ with $\mathbf{r}=\mathbf{x}_{2}-\mathbf{x}_{1}$ as the relative distance between the localized spins. Note that we have assumed that the Green's function is spin independent in the above derivation. An additional term such as a Dzyaloshinskii-Moriya type of interaction can emerge in spin-orbital coupled systems where the Green's functions depend on spin [28,29]. It is also desirable to generalize Eq. (21) to $\operatorname{SU}(N)$ RKKY interactions due to the remarkable progress in experiments [30,31].

The two possibilities, $\alpha(\mathbf{r})>0[\alpha(\mathbf{r})<0]$, correspond to a ferromagnetic (antiferromagnetic) alignment of magnetic moments. Consider two magnetic moments (spins) that are embedded in a system with chiral symmetry, and have definite chiralities $\chi_{1}$ and $\chi_{2}$, respectively. The susceptibility can then be written as

$$
\alpha^{\chi_{1} \chi_{2}}=-\frac{1}{2 \beta} \sum_{n} \mathcal{G}^{\chi_{1} \chi_{2}}\left(i \omega_{n}, \mathbf{r}\right) \mathcal{G}^{\chi_{2} \chi_{1}}\left(i \omega_{n},-\mathbf{r}\right) .
$$

Based on the symmetry properties of Green's functions [see Eqs. (14a) and (14b)], we can verify that $\alpha^{\chi \chi}>0$ and $\alpha^{\chi \bar{\chi}}<$ 0 . As a result, magnetic moments with the same (opposite) chirality favor a ferromagnetic (antiferromagnetic) alignment. This applies to all the examples discussed in the previous sections. Note that our theorem exhibits two major differences compared to the theorem concerning the sign of RKKY interactions on noninteracting bipartite lattices [34,35]. First, our 
theorem has a broader scope of application, as the bipartite model is only a specific system preserving chiral symmetry. Second, our theorem can be applied to interacting models as long as chiral symmetry is present. This indicates the possibility of chiral symmetry breaking in the previous calculations, which showed that the sign of the RKKY interactions can be modified by electronic interactions, edge states, strains, or flat bands [36].

Concluding remarks. We have established a universal theorem regarding the sign of fermion-mediated interactions in systems with chiral symmetry. We have demonstrated the strength of the theorem by considering multiple examples ranging from noninteracting models to weakly correlated systems. Without involving any spatial symmetry, the theorem is robust to disorders and defects. Furthermore, our theorem suggests another route to probe chiral symmetry breaking via fermion-mediated interactions: When additional parameters break chiral symmetry, the sign of fermion-mediated interactions will not be definite, and will oscillate with respect to these parameters. In the future, it will be interesting to investigate how other symmetries (e.g., crystalline symmetries) constrain fermion-mediated interactions. It would also be desirable to study the sign of two-boson-mediated interactions in systems with chiral symmetry [37].

Acknowledgments. We thank F. Wilczek for his encouragement and support, and E. Bergholtz, L. Liang, Y. Kedem, and especially T. H. Hansson for indispensable clarifying discussions. We thank K. Dunnett for her proofreading of the manuscript. This work was supported by the Swedish Research Council under Contract No. 335-2014-7424.
[1] A. Bulgac and A. Wirzba, Phys. Rev. Lett. 87, 120404 (2001); P. Sundberg and R. L. Jaffe, Ann. Phys. (N. Y.) 309, 442 (2004); D. Zhabinskaya, J. M. Kinder, and E. J. Mele, Phys. Rev. A 78, 060103(R) (2008).

[2] An excellent introduction can be found in A. Zee, Quantum Field Theory in a Nutshell (Orient Longman, Princeton, NJ, 2005).

[3] T. B. Grimley, Proc. Phys. Soc. 90, 751 (1967); 92, 776 (1967); T. L. Einstein and J. R. Schrieffer, Phys. Rev. B 7, 3629 (1973); N. R. Burke, Surf. Sci. 58, 349 (1976); K. H. Lau and W. Kohn, ibid. 75, 69 (1978).

[4] For recent experiments, see J. Repp, F. Moresco, G. Meyer, K.H. Rieder, P. Hyldgaard, and M. Persson, Phys. Rev. Lett. 85, 2981 (2000); F. Silly, M. Pivetta, M. Ternes, F. Patthey, J. P. Pelz, and W. D. Schneider, ibid. 92, 016101 (2004); M. Ternes, C. Weber, M. Pivetta, F. Patthey, J. P. Pelz, T. Giamarchi, F. Mila, and W.-D. Schneider, ibid. 93, 146805 (2004).

[5] V. V. Cheianov and V. I. Fal'ko, Phys. Rev. Lett. 97, 226801 (2006); V. V. Cheianov, O. Syljuåsen, B. L. Altshuler, and V. Fal'ko, Phys. Rev. B 80, 233409 (2009); V. V. Cheianov, O. Syljuåsen, B. L. Altshuler, and V. I. Fal'ko, Europhys. Lett. 89, 56003 (2010); D. A. Abanin, A. V. Shytov, and L. S. Levitov, Phys. Rev. Lett. 105, 086802 (2010).

[6] M. A. Ruderman and C. Kittel, Phys. Rev. 96, 99 (1954); T. Kasuya, Prog. Theor. Phys. 16, 45 (1956); K. Yosida, Phys. Rev. 106, 893 (1957).

[7] M. M. Parish, B. Mihaila, B. D. Simons, and P. B. Littlewood, Phys. Rev. Lett. 94, 240402 (2005); J. M. Acton, M. M. Parish, and B. D. Simons, Phys. Rev. A 71, 063606 (2005); S. T. Chui and V. N. Ryzhov, ibid. 69, 043607 (2004); D. H. Santamore and E. Timmermans, ibid. 78, 013619 (2008); S. De and I. B. Spielman, Appl. Phys. B 114, 527 (2014); J. J. Kinnunen and G. M. Bruun, Phys. Rev. A 91, 041605(R) (2015); D. Suchet, Z. Wu, F. Chevy, and G. M. Bruun, ibid. 95, 043643 (2017).

[8] B. DeSalvo, K. Patel, G. Cai, and C. Chin, Nature (London) 568, 61 (2019); H. Edri, B. Raz, N. Matzliah, N. Davidson, and R. Ozeri, Phys. Rev. Lett. 124, 163401 (2020).

[9] A. Bulgac and P. Magierski, Nucl. Phys. A 383, 695 (2001), and references therein; Y. Yu, A. Bulgac, and P. Magierski, Phys. Rev. Lett. 84, 412 (2000).
[10] A. Chodos, R. L. Jaffe, K. Johnson, C. B. Thorn, and V. F. Weisskopf, Phys. Rev. D 9, 3471 (1974); G. Neergaard and J. Madsen, ibid. 62, 034005 (2000), and references therein; K. Hattori, K. Itakura, S. Ozaki, and S. Yasui, ibid. 92, 065003 (2015).

[11] A. V. Shytov, D. A. Abanin, and L. S. Levitov, Phys. Rev. Lett. 103, 016806 (2009).

[12] S. LeBohec, J. Talbot, and E. G. Mishchenko, Phys. Rev. B 89, 045433 (2014); M. Agarwal and E. G. Mishchenko, ibid. 95, 075411 (2017).

[13] A. Recati, J. N. Fuchs, C. S. Peça, and W. Zwerger, Phys. Rev. A 72, 023616 (2005); E. B. Kolomeisky, J. P. Straley, and M. Timmins, ibid. 78, 022104 (2008); A. Mering and M. Fleischhauer, ibid. 81, 011603(R) (2010); A. Flachi, Phys. Rev. Lett. 110, 060401 (2013); A. Flachi, M. Nitta, S. Takada, and R. Yoshii, ibid. 119, 031601 (2017).

[14] H. P. Büchler and G. Blatter, Phys. Rev. Lett. 91, 130404 (2003); P. P. Orth, D. L. Bergman, and K. Le Hur, Phys. Rev. A 80, 023624 (2009).

[15] V. Gurarie, Phys. Rev. B 83, 085426 (2011); Z. Wang and S.-C. Zhang, ibid. 86, 165116 (2012).

[16] S. Ryu, A. Schnyder, A. Furusaki, and A. Ludwig, New J. Phys. 12, 065010 (2010); C.-K. Chiu, J. C. Y. Teo, A. P. Schnyder, and S. Ryu, Rev. Mod. Phys. 88, 035005 (2016).

[17] C. D. Fosco and E. L. Losada, Phys. Rev. D 78, 025017 (2008); S. Bellucci and A. A. Saharian, ibid. 80, 105003 (2009).

[18] O. Kenneth and I. Klich, Phys. Rev. Lett. 97, 160401 (2006); Phys. Rev. B 78, 014103 (2008); T. Emig, N. Graham, R. L. Jaffe, and M. Kardar, Phys. Rev. Lett. 99, 170403 (2007); S. J. Rahi, T. Emig, N. Graham, R. L. Jaffe, and M. Kardar, Phys. Rev. D 80, 085021 (2009).

[19] Q.-D. Jiang and F. Wilczek, Phys. Rev. B 99, 125403 (2019).

[20] J. M. Byers, M. E. Flatté, and D. J. Scalapino, Phys. Rev. Lett. 71, 3363 (1993); M. I. Salkola, A. V. Balatsky, and D. J. Scalapino, ibid. 77, 1841 (1996); W. Ziegler, D. Poilblanc, R. Preuss, W. Hanke, and D. J. Scalapino, Phys. Rev. B 53, 8704 (1996); C. Bena and S. A. Kivelson, ibid. 72, 125432 (2005).

[21] Due to the properties of the $\log$ function, $\log (1-x)<0$ for $x>0$, and $\log (1-x)>0$ for $x<0$. 
[22] A. H. Castro Neto, F. Guinea, N. M. R. Peres, K. S. Novoselov, and A. K. Geim, Rev. Mod. Phys. 81, 109 (2009).

[23] T. O. Wehling, S. Yuan, A. I. Lichtenstein, A. K. Geim, and M. I. Katsnelson, Phys. Rev. Lett. 105, 056802 (2010); T. O. Wehling, M. I. Katsnelson, and A. I. Lichtenstein, Chem. Phys. Lett. 476, 125 (2009).

[24] E. McCann and V. I. Fal'ko, Phys. Rev. Lett. 96, 086805 (2006); J. Nilsson, A. H. Castro Neto, N. M. R. Peres, and F. Guinea, Phys. Rev. B 73, 214418 (2006); M. Koshino, T. Nakanishi, and T. Ando, ibid. 82, 205436 (2010).

[25] G. Tarnopolsky, A. J. Kruchkov, and A. Vishwanath, Phys. Rev. Lett. 122, 106405 (2019); P. San-Jose, J. González, and F. Guinea, ibid. 108, 216802 (2012); J. Wang, Y. Zheng, A. J. Millis, and J. Cano, arXiv:2010.03589.

[26] M. S. Foster and A. W. W. Ludwig, Phys. Rev. B 77, 165108 (2008).

[27] J. Verbaarschot, Phys. Rev. Lett. 72, 2531 (1994); S. Yasui and K. Sudoh, Phys. Rev. C 88, 015201 (2013);

[28] F. Ye, G. H. Ding, H. Zhai, and Z. B. Su, Europhys. Lett. 90, 47001 (2010); J.-J. Zhu, D.-X. Yao, S.-C. Zhang, and K. Chang, Phys. Rev. Lett. 106, 097201 (2011); A. A. Zyuzin and D. Loss, Phys. Rev. B 90, 125443 (2014).

[29] D. K. Efimkin and V. Galitski, Phys. Rev. B 89, 115431 (2014); H.-R. Chang, J. Zhou, S.-X. Wang, W.-Y. Shan, and D. Xiao, ibid. 92, 241103(R) (2015); V. Kaladzhyan, A. A. Zyuzin, and P. Simon, ibid. 99, 165302 (2019).

[30] A. V. Gorshkov, M. Hermele, V. Gurarie, C. Xu, P. S. Julienne, J. Ye, P. Zoller, E. Demler, M. D. Lukin, and A. M. Rey, Nat. Phys. 6, 289 (2010); A. J. Keller, S. Amasha, I. Weymann,
C. P. Moca, I. G. Rau, J. A. Katine, H. Shtrikman, G. Zaránd, and D. Goldhaber-Gordon, ibid. 10, 145 (2014); M. Ferrier, T. Arakawa, T. Hata, R. Fujiwara, R. Delagrange, R. Deblock, Y. Teratani, R. Sakano, A. Oguri, and K. Kobayashi, Phys. Rev. Lett. 118, 196803 (2017); L. Riegger, N. Darkwah Oppong, M. Höfer, D. R. Fernandes, I. Bloch, and S. Fölling, ibid. 120, 143601 (2018).

[31] See Supplemental Material at http://link.aps.org/supplemental/ 10.1103/PhysRevB.103.L121107 for extended discussions, which includes Refs. [32,33].

[32] B. Coqblin and J. R. Schrieffer, Phys. Rev. 185, 847 (1969); M. Hermele, V. Gurarie, and A. M. Rey, Phys. Rev. Lett. 103, 135301 (2009).

[33] M. Hermele and V. Gurarie, Phys. Rev. B 84, 174441 (2011); M. Raczkowski and F. F. Assaad, Phys. Rev. Res. 2, 013276 (2020).

[34] S. Saremi, Phys. Rev. B 76, 184430 (2007); L. Brey, H. A. Fertig, and S. Das Sarma, Phys. Rev. Lett. 99, 116802 (2007).

[35] E. Kogan, Phys. Rev. B 84, 115119 (2011).

[36] A. M. Black-Schaffer, Phys. Rev. B 82, 073409 (2010); 81, 205416 (2010); J. E. Bunder and H.-H. Lin, ibid. 80, 153414 (2009); P. D. Gorman, J. M. Duffy, M. S. Ferreira, and S. R. Power, ibid. 88, 085405 (2013); D. O. Oriekhov and V. P. Gusynin, ibid. 101, 235162 (2020).

[37] For example, we have shown that anomalous Casimir interactions can be induced by a chiral-symmetry-breaking medium [19] or a time-reversal-symmetry-breaking medium [see Q.-D. Jiang and F. Wilczek, Phys. Rev. B 99, 201104(R) (2019); 99, 165402 (2019)]. 